\title{
THE STELLAR POPULATIONS IN \\ LOCAL GROUP DWARF GALAXIES
}

\author{
ABHIJIT SAHA \\ Space Telescope Science Institute \\ 3700 San Martin Drive, Baltimore MD 21218, USA \\ (saha@stsci.edu)
}

\section{Introduction}

The aim of the study of the populations in a stellar system is to understand and be able to describe the stellar content of a system in terms of physical parameters such as the age, star formation history, chemical enrichment history, initial mass function (IMF), environment, and dynamical history of the system. This is done given an understanding of stellar evolution and the ability to express the outcome in "observer parameters", particularly a color-magnitude diagram (CMD), kinematics, and metallicity. From this perspective, the simplest systems are the galactic clusters and the globular clusters, where all the component stars are coeval and of the same metallicity. The current state of knowledge for these are discussed by others in this conference. We proceed to the next level of complexity (where metallicities are not necessarily all the same, and nor are the stars all coeval), and try to decompose their stellar content, particularly in terms of star formation rate and metallicity. In this regard the two classes of objects that come to mind are the dwarf spheroidals, and the dwarf irregulars. Both these classes of objects are more massive than the open clusters and globular clusters, and show evidence of complexities in their star formation histories, without being so convolved as to make such a study intractable. As we shall see, recent studies along these lines have presented some puzzling problems. Moreover, these are the smallest independent galaxies, and the study of star formation in these is likely to shed light on the history and formation of larger and more complex galaxies.

The availability of relatively large format and efficient high quality CCD detectors, and the development of software tools to perform photometry in 
crowded fields has opened the door to study the stars in such galaxies via their CMDs. The discussion here does not include the dwarf ellipticals M32 and NGC 147, 185 and 205, since they are discussed by others in this conference.

The known dwarf spheroidals, which have $-14 \leq M_{V} \leq-8$, are mostly clustered around the Galaxy and M31. They contain stars that are $\geq 1 \mathrm{Gyr}$ old. The most luminous stars in them are red giants and asymptotic giant branch (AGB) stars, and sometimes luminous carbon stars. In contrast, the dwarf irregulars show no particular preference or clustering within the Local Group. They contain luminous young stars: both blue and red supergiants that indicate recent or ongoing star formation. They have $M_{V} \leq-11$. The thrust of this presentation is to highlight recent work on both these types of objects: to show the variety in star formation history that is seen even within each of these classes of objects on the one hand, and also to outline the elements that are common across the two apparently different types of objects.

\section{The Dwarf Spheroidals}

A recent review of the dwarf spheroidal galaxies has been given by van den Bergh (1994). This is also an excellent lead in to the past literature on these objects. The most used discriminant for stellar populations in these objects is the CMD. A CMD that shows a turnoff 3.5 mags fainter than the horizontal branch (HB) is necessary and sufficient evidence for stars that are older than 10 Gyr. For the purpose of this discussion we shall call these "old" stars. The presence of an extended HB and/or the presence of significant numbers of RR Lyrae stars is sufficient but not necessary (due to the "second parameter" variation) evidence for the presence of old stars. Globular clusters in our Galaxy exemplify such a population. The CMD of the Draco dwarf galaxy (Carney and Seitzer, 1986), for example, is indistinguishable from the CMD of the metal poor globular cluster M92. Other examples of dwarf spheroidals made up entirely of old metal poor stars are the Sculptor and Ursa Minor dwarfs.

Necessary but not sufficient evidence of a stellar population younger than $10 \mathrm{Gyr}$ is the presence of a "clump" or "stub" at the base of the giant branch in lieu of an extended HB. Necessary and sufficient evidence is that the main sequence turnoff is bright: less than 3 mag fainter than the clump. If, in addition, the brightest stars have $M_{I}$ fainter than -4 , it rules out the presence of stars younger than 1 Gyr. For the purpose of this discussion we shall call these "intermediate age" stars, spanning an age range from 1 to $10 \mathrm{Gyr}$. The presence of luminous carbon stars is also an indication of the presence of intermediate age stars. The CMD obtained 
by Mould and Aaronson (1983) for the Carina dwarf spheroidal galaxy showed just such a population. The small region of that galaxy sampled by them showed a clump rather than an extended horizontal branch, but more significantly, showed a very bright turnoff only 2 mags fainter than the clump: incontrovertible evidence for stars only 6 Gyrs old. Subsequently, intermediate age stars were also seen in the Fornax (Buonanno et al., 1985) and Leo I (Demers et al., 1993) dwarf spheroidals, showing that such ages are quite common in these objects.

It was not until very recently that observed CMDs could go deep enough to examine whether in addition to the intermediate age stars there were also old stars from an initial burst of star formation. The only hint at their presence was from the large number of RR Lyrae stars found in the Carina dwarf (Saha et al., 1986). The spectacular new CMD of the Carina dwarf galaxy by Smecker-Hane et al. (1994) (shown also in Hesser's presentation at this conference), shows both, a thinly populated extended HB, as well as turnoff stars spanning a range in brightness. The faintest turnoff stars are in fact about 3.5 mag fainter than the HB, indicating that star formation in this galaxy has spanned several Gyr, beginning well before 10 Gyr ago. A CMD for the Fornax dwarf obtained by Beauchamp et al. (1994) shows a range of turnoffs beginning 2-3 Gyr ago. Turnoff stars are seen all the way to the faint limit of the CMD, showing stars up to $7 \mathrm{Gyr}$ old. Unfortunately the CMD is not sufficiently deep to probe the turnoff from old stars.

One aspect in which the Carina and Fornax CMDs differ is that the former shows a very narrow giant branch, indicating little if any enrichment in metallicity, despite the large range of ages. Fornax however shows a metallicity range $-2.0 \leq[\mathrm{Fe} / \mathrm{H}] \leq-1.0$.

The deep CMD of Leo I (Olszewski et al, 1995) shows turnoff stars only 1.2 mags below the clump, indicating ages as young as 1.5 Gyrs. While there is no extended HB, there are turnoff stars nearly 3.5 mags fainter than the clump: the fitted isochrones indicate the oldest age to be $12 \mathrm{Gyr}$. Again, this is spectacular evidence of star formation beginning more than 10 Gyr ago, but continuing till rather recently.

An interesting hypothesis is presented by van den Bergh (1994), where it is suggested that those dwarf spheroidals that are closer to the Galaxy (say within $100 \mathrm{kpc}$ ) have only old stars (Ursa Minor, Sculptor, Draco and Sextans), whereas those that are farther away have more recent star formation history. The implication is that proximity to the Galaxy has caused the cessation of star formation in the nearer dwarfs, perhaps by sweeping out the gas. Carina is a notable exception, since it is among the nearer galaxies, but has had an extended star formation history. At the other end, a recent CMD obtained with HST for the Tucana dwarf (Walker, 1994), which is $800 \mathrm{kpc}$ away, and apparently associated with neither the 
Galaxy nor M31, shows a well populated extended HB. No turnoff is visible up to 1.5 mags below the HB (unfortunately one cannot probe deeper even with HST), and the well populated HB indicates that the majority of the stars are "old". This also is a counter example to van den Bergh's thought provoking hypothesis. One is left to ponder the following questions:

- Is the apparent distribution of dwarf spheroidals as clumped around the Galaxy and M31 real or the result of bias arising from a difficulty of finding these inconspicuous diffuse objects at large distances? The existence of the Tucana dwarf is an example in point.

- How is it that while we see multiple epochs of star formation in some of the dwarfs, no significant gas has been detected in any of these objects?

\section{The Dwarf Irregulars}

These objects are distributed in the Local Group without any apparent conglomeration around M31 or the Galaxy. Probes of their stellar population include CMDs, and some spectroscopy of the HII regions that delineate metallicity of the youngest associations and star forming regions.

\subsection{THE YOUNG STARS}

The CMD provides a very useful tool for probing the young stellar content in these objects and deciphering the recent star formation history. The technique is to compare the observed CMD to a model CMD constructed from physical parameters like metallicity, initial mass function, star formation rate. Stellar evolution tracks for these parameters can then be used to generate a synthetic H-R diagram, which in turn is converted to the observers CMD plane by using model atmospheres and filter transmission functions. The pioneering attempt with this approach is given in Greggio et al. (1993). Here they inspect the observed and synthetic CMDs side by side, after convolving the latter with some appropriate model for the errors and crowding and blending effects. They are able to achieve good agreement for the galaxy NGC 3109, for which the distance is well determined from Cepheids. Their attempt at DDO 210 shows the difficulty of applying this approach when the distance is not known in advance, since the uncertainty in distance introduces severe degeneracy in such a procedure.

Hoessel and Saha have undertaken a program to measure distances to several local group dwarf irregulars by discovering and measuring Cepheids. This project is well underway, and has yielded distances to Pegasus (Hoessel et al., 1990), Leo A (Hoessel et al., 1994) GR 8 (Tolstoy et al., 1995) and IC 10 (Saha and Hoessel, 1995). An improved method of matching observed CMDs to synthetic ones has been devised, which utilizes maximum likelihood techniques to compare CMDs, explicitly accounts for observa- 
tional errors on a star by star basis, considers detection incompleteness as a function of magnitude, and attempts to model "confusion" noise and blending frequency to account for crowding. When combined with Cepheid distances, this proves to be a powerful method of determining the recent star formation history. A poster paper by Eline Tolstoy at this meeting illustrates this technique for Leo A and GR 8.

\subsection{THE OLD AND INTERMEDIATE AGE STARS}

The old and intermediate age stars in these objects are hard to study, since they are buried in the glare of the bright supergiants from the younger population. The brightest red giants representing stars older than $1 \mathrm{Gyr}$ are expected, however, to be more diffusely distributed, so deep images of the outer regions of these galaxies can reveal these denizens in large numbers past a critical faint limit corresponding to the tip of the red giant branch. Baade (1944) was the first to exploit this method to resolve the bulge of M31 revealing the Population II giants.

Such resolution into the so called "Baade sheet" (of stars), is proof of at least an intermediate age population, if not a population with age exceeding 10 Gyrs. Though Baade knew of the extended elliptical halo of the dwarf IC 1613, the discovery of RR Lyrae stars (Saha et al., 1992) established the existence of old stars in this object. The outer regions of IC 10 have also now been resolved into the "Baade sheet", although the existence of $10 \mathrm{Gyr}$ and older stars has not yet been established. Even in the star-burst galaxy NGC 5253, which is in the M81 group, HST observations have revealed the existence of an old/intermediate age population by resolution into the "Baade sheet" (Saha et al., 1995).

The point to consider is that whenever data of adequate nature are examined, the presence of old or intermediate age objects is evident. It is interesting to examine this hypothesis on a larger sample. The repaired HST (which has a limiting magnitude of $V \approx 27.5 \mathrm{mag}$ ) is able to search for old and intermediate stars in most Local Group dwarf irregulars (which have typical distance modulii of 25 to 26 ) via the following routes:

$-V \approx 25$ mag gets the tip of the red giant branch for all Local Group objects, and

$-V \approx 27$ mag gets the horizontal branch morphology and/or RR Lyrae stars for many Local Group dwarf irregular galaxies.

\section{Summary}

We see that recent work on the dwarf spheroidal galaxies shows, in many instances, evidence for extended periods of star formation. None of them have detectable amounts of gas left in them, and show no evidence of star 
formation in the recent past (within $1 \mathrm{Gyr}$ ). They are apparently clustered around the Galaxy and M31, though it is unclear whether this is a selection effect. On the other hand, the dwarf irregulars, which do have gas, and do have ongoing star formation, also show evidence of intermediate and old stars whenever data of sufficient quality to detect these are available. It is thus tempting to think of a connection between these 2 types of objects, and to ascribe their distinction to the presence or absence of gas and ongoing star formation. To examine this it is necessary to pursue two lines of investigation: to look for more dwarf spheroidals like the Tucana dwarf that are unconnected to the Galaxy or M31 to test whether they are distributed like the (easier to detect) dwarf irregulars, and on the other hand to examine whether the presence of old stars in the dwarf irregulars is universal. Additionally, a more extensive comparison of the internal dynamics of these two classes of objects can be a test of commonality.

\section{References}

Baade, W., 1944, Ap.J. 100, 147

Beauchamp, D., Hardy, E., Suntzeff, N. B. and Zinn R., 1984, A.J., submitted

Buonanno, R., et al., 1985, A. \& A. 152, 65

Carney, B. and Seitzer, P., 1986, A. J. 92, 23

Demers, S., et al., 1993, M. N.R. A. S. 261, 657

Greggio, L., Marconi, G., Tosi, M. and Focardi, P., 1993, A. J. 105, 894

Hoessel, J. G., Abbott, M., Saha, A., Mossman, A. and Danielson, G. E., 1990, A. J. 100, 1151

Hoessel, J. G., Saha, A., Krist, J. and Danielson, G. E., 1994, A. J. 108, 645

Mould, J. R. and Aaronson, M., 1983, Ap. J. 273, 530

Olszewski, E. W., Mateo, M., Saha, A., Lee, M.-G., Hodge, P., Keane, M., Suntzeff, N., Freedman, W. and Thompson, I., 1995 (in preparation)

Saha, A., Monet, D. G. and Seitzer, P., 1986, A. J. 92, 302

Saha, A., Freedman, W., Hoessel, J. G. and Mossman, A., 1992, A. J. 104, 1072

Saha, A. and Hoessel, J. G., 1995, (in preparation)

Saha, A., Sandage, A., Labhardt, L., Schwengeler, H., Tammann, T. A., Panagia, N. and Macchetto, F. D., 1995, Ap. J., (in press)

Smecker-Hane, T. A., Stetson, P. B. and Hesser, J. E., 1994, private communication

Tolstoy, E., Saha, A., Hoessel, J. G. and Danielson, G. E., 1995, A. J., in press

van den Bergh, S., 1994, Ap. J. 428, 617

Walker, A., 1994, private communication

GERHARDT: Concerning the evidence you showed for a star formation episode in Leo I as recent as 1.5 Gyrs ago: is it possible to estimate the mass of gas transformed into stars in this event, its duration, and the fraction of Leo I's mass that might have been lost?

SAHA: The CMD shown represents only a small fraction of the total observation set, so the statistics will improve a lot, and it may be possible to say something interesting about the duration of the final star formation episode, if in fact it was episodic and not continuous. To get the mass that went into it, you need to know the mass function at the faint end, so it may not be very easy to get. 